\title{
FORMULATION AND EVALUATION OF SUSTAINED RELEASE MATRIX TABLETS OF NATEGLINIDE
}

\author{
K.B. Patel", J.R.Vyas, U.M. Upadhyay
}

Sigma Institute of Pharmacy, Bakrol, Vadodara, Gujarat, India

“Corresponding Author's Email: krishupatel22@gmail.com, Contact No: - +91-9974763192

Received 04 Aug 2015; Review Completed 25 Aug 2015; Accepted 26 August 2015, Available online 15 Sep 2015

\begin{abstract}
The objective of this work was to prepare and evaluate oral sustained release matrix tablet of Nateglinide and to study the effect of proportion of wax and addition of release liner on in-vitro release of drug.The prepared tablets were evaluated for pre and post compression parameters. Stability study of the promising formulation was also performed. The matrix tablets were prepared by Direct compression, Co-processed \& melt granulation, method using wax in concentration $25 \%, 35 \%$ \& $45 \%$ and evaluated for on in-vitro drug release using Compritol \& Precirol. No interactions were found between drug and excipients. Formulation containing 25\% Precirol F13 shows releases up to 12 hours. Tablets with release characteristics offers critical advantages such as site specificity with improved absorption and efficacy etc.
\end{abstract}

Keywords: Nateglinide, Sustained release, Compritol 888, Precirol ATO 5, Melt Granulation, Direct compression, Coprocessing

\section{INTRODUCTION}

A sustained-release dosage form is defined as "any drug or dosage form modification thatprolongs the therapeutic activity of the drug" 1 . Development of oral sustained release (SR) tablets of highly water soluble drugs or bioactives has always been a challenge and therefore, opportunity for formulation scientist. Most of these drugs if not formulated properly, may be released at a faster rate resulting in exceeding the maximum therapeutic levels and hence will lead to toxic side effects. Sustained delivery of such drugs ensures improved drug delivery and patient compliance, greater safety and efficacy, desired release kinetics and helps in maintaining the plasma drug concentration within the therapeutic window for extended period of time ${ }^{2,3}$ Lipids like glycerides are a family of excipients which have generated considerable interest in the preparation of oral dosage forms. Some glycerides such as Compritol ATO 888 (glyceryl behenate), Precirol ATO 5 (glyceryl palmitostearate) can be used for the preparation of sustained release dosageforms ${ }^{5}$. The esterification of glycerol by long chain fatty acid gives them a pronounced hydrophobic character with a low HLB value of $2^{6}$. Several techniques including melt granulation ${ }^{7}$, melt pelletization ${ }^{8}$, hot melt extrusion ${ }^{9}$ and hot melt coating ${ }^{10}$ have been used to obtain sustained release dosage forms from glycerides-based formulations.

Melt granulation (MG) is a solvent-free process which involves the use of a substance that melts at a relatively low temperature. This substance can be added in the molten form over the substrate or in the solid form, which is then heated above its melting point. The substance acts as a liquid binding agent, and the technique does not require the use of organic solvents.
Moreover, in melt granulation drying is not necessary and thus, the process is less consuming in terms of time and energy compared to other methods ${ }^{4}$. Sustained release matrix tablets have been produced with Compritol ATO 888 \& Precirolby various methods including $\mathrm{MG}^{11}$, Co-processing and direct compression (DC) $)^{12}$

Nateglinide, $N$-(trans-4-isopropylcyclohexylcarbonyl)D-phenylalanine] is a phenylalanine derivative lackingeither a sulfonylurea or benzamido moiety, which can restore the physical insulin secretion pattern lost in type 2 diabetes mellitus patients with postprandial hyperglycemia. Pharmacologically, nateglinide reportedly acts on pancreatic cells via the closure of the adenosine triphosphate-sensitive potassium channels by binding to sulphonylurea receptor subunits causingcell depolarization, calcium influx, and insulin release. The drug has a rapidonset and short duration of insulinotropic action $\left(\mathrm{t}^{\mathrm{t}} / 2\right.$ is $\_1.5$ hours and Tmax is_0.5-1.0 hour after dosing). ${ }^{13,14}$

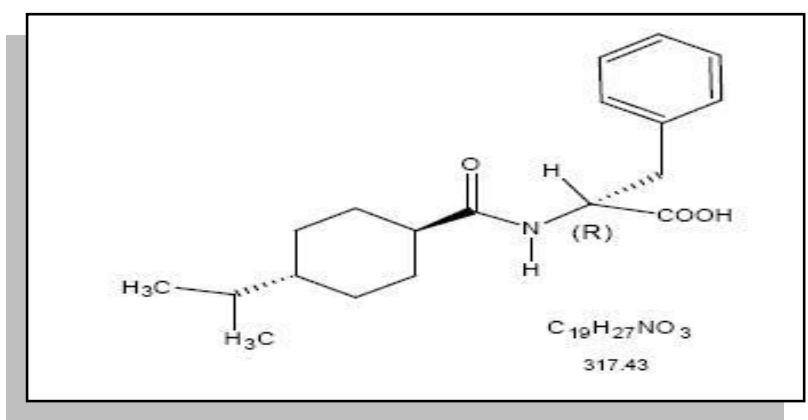

Figure 1: Nateglinide structure 


\section{MATERIALS AND METHODS}

\section{Materials}

Nateglinide was a gift from Pure chem, Bharuch, India while Compritol ATO 888 and Precirolwere obtained free of charge from Gattefosse SAS India. Di-Calcium phosphate, magnesium stearate, Aerosil 200, concentrated hydrochloric acid, sodium hydroxide and potassium dihydrogen phosphate were purchased from SD Fine chemicals, Mumbai, India. Distilled water (D.W.)was prepared freshly whenever required. Other chemicals and reagents used were of analytical grade.

\section{Methods}

Preparation of Nateglinide matrix tablets

Preparation of wax matrix tablets was done by three methods: Melt granulation, Co-processing and Direct compression as follows.

\section{Identification of drug:}

The obtained sample was examined by infrared absorption spectral analysis and was compared with the reference standard IR spectrum of API. Also UV spectrum was taken to identify the compound based on $\lambda_{\text {max }}$ and peak shape.

\section{Organoleptic characteristics:}

The color, odour and taste of the drug were characterized and recorded using descriptive terminology.

\section{Solubility of drug:}

The solubility of Drug was determined as per BCS. The solubility was checked in $250 \mathrm{ml}$ of $0.01 \mathrm{~N} \mathrm{HCl}$ with $0.5 \%$ SLS and buffers within $\mathrm{pH}$ range $2-8$. The highest amount of dose was accurately weighed and transferred in individual volumetric flask containing different solutions and sonicate for 30 minutes. Interpretation was done based on BCS classification which states that drug is BCS class II.

\section{Pre compression parameters :}

a) Angle of repose : Angle of repose of the granules will be measured by the fixed height method.

$\theta=\tan ^{-1} \mathrm{~h} / \mathrm{r} \theta=$ Angle of repose

$$
\begin{aligned}
& \mathrm{h}=\text { Height of powder heap } \\
& \mathrm{r}=\text { Radius of the powder cone }
\end{aligned}
$$

b) Bulk density : The powder sample equivalent to $10 \mathrm{~g}$ will be accurately weighed, will fill in a $50 \mathrm{ml}$ graduated cylinder, the powder will be levelled \& the unsettled volume (Vi) will be noted. The bulk density will be calculated in $\mathrm{g} / \mathrm{cm} 3$ by the formula.

$$
\begin{gathered}
\rho_{\mathrm{i}}=\mathrm{m} / \mathrm{V}_{\mathrm{i}} \mathrm{m}=\text { mass of the blend } \\
\mathrm{V}_{\mathrm{i}}=\text { untapped volume }
\end{gathered}
$$

c) Tapped density :

$$
\rho_{\mathrm{t}}=\mathrm{m} / \mathrm{V}_{\mathrm{t}} \quad \mathrm{V}_{\mathrm{t}} \text { is tapped volume }
$$

d) Hausner's Ratio : It is measurement of frictional resistance of the drug. The ideal range should be
1.2-1.5. It will be determined by the ratio of tapped density and bulk density.

Tapped density/bulk density

e) Carr's index : Based on the bulk density \& tapped density, the percentage compressibility of the granules will be computed using the Carr's compressibility index by the formula,

(Tapped density-bulk density)*100/Tapped density

Post compression parameters:

a) Weight Variation : Will be done as per USP

b) Thickness : Will be Measured by Vernier Calipers

c) Hardness : Will be Measured by Monsanto Hardness Tester

d) Friability : Will be Measured by Roche friabilator

$$
\begin{aligned}
& \% \text { Friability }=\left(\mathrm{W}_{1}-\mathrm{W}_{2} / \mathrm{W}_{2}\right) * 100 \\
& \mathrm{~W}_{1}=\text { weight of tablets before test } \\
& \mathrm{W}_{2}=\text { weight of tablets after test }
\end{aligned}
$$

\section{e) In-Vitro dissolution study:}

An in-vitro dissolution study will be carried out in

- Dissolution medium: $0.5 \%$ sodium lauryl sulphate (SLS) in $900 \mathrm{ml}$ of Phosphate buffer (pH 6.8) with type II paddle.

- Temperature: $37 \pm 0.5^{\circ} \mathrm{C}$

- $\quad$ Stirring speed of Paddle:50rpm

- $\quad$ Time Point(hr): 1,2,3,4,5,6,7,8,9,10,11,12

- Sample amount: $5 \mathrm{ml}$

- Volume: $900 \mathrm{ml}$

\section{Method of preparation:}

Melt granulation method: The waxes(Compritol 888 ATO\& Precirol ATO 5) was melted in a porcelain dish over a water bath maintained at $75-80^{\circ} \mathrm{C}$ for $3 \mathrm{~min}$ and Nateglinide was gradually added with continuous stirring until uniformly mixed. The molten mixture was allowed to cool and solidify at room temperature crushed in a mortar and passed through a $40 \#$ sieve. The granules were compressed into flat-faced tablet using multi-station rotary tablet compression machine (Krishna engineering Pvt. Ltd. India) at a constant compression force.

Evaluation of drug - Excipient interaction: The pure drug, wax and the matrix tablet formulation were subjected to IR spectroscopy using FT-IR spectrophotometer. Their spectra were obtained over the wave number range of $4000-400 \mathrm{~cm}^{-1}$.

In-vitro drug release: In vitro release studies were conducted using USP type II paddle apparatus (VDA$6 \mathrm{D}$ USP Std -VEEGO) run at $50 \mathrm{rpm}$. The buffer was kept at thermostatically controlled temperature of $37 \pm 0.5^{\circ} \mathrm{C}$. The test was carried out in $900 \mathrm{ml}$ of $0.01 \mathrm{M}$ $\mathrm{HCl}$ for $2 \mathrm{~h}$ and then replaced with phosphate buffer $(\mathrm{pH}$ 6.8) as the dissolution medium for another $10 \mathrm{~h}$. The $\mathrm{pH}$ change of medium was effected by adding $4.32 \mathrm{~g}$ of sodium hydroxide and $6.08 \mathrm{~g}$ of potassium 
dihydrogen phosphate dissolved in $5 \mathrm{ml}$ water to the previous acidic medium $(0.1 \mathrm{M} \mathrm{HCl})(17)$. Five milliliters samples were withdrawn at the time intervals of $1,2,3,4,5,6 \ldots .12$ and replaced with equal volume of fresh dissolution medium. The samples were filtered through $0.45 \mu \mathrm{m}$ filter and analyzed for drug content at $218 \mathrm{~nm}$ by UV spectrophotometer.

Formulation:

Table 1: Identical formulation table for all batches

\begin{tabular}{|c|c|c|c|c|c|c|}
\hline Polymer used & \multicolumn{3}{|c|}{ Precirol ATO 5 } & \multicolumn{3}{c|}{ Compretol 888 } \\
\hline & $\mathbf{2 5 \%}$ & $\mathbf{3 5 \%}$ & $\mathbf{4 5 \%}$ & $\mathbf{2 5 \%}$ & $\mathbf{3 5 \%}$ & $\mathbf{4 5 \%}$ \\
\hline Drug (NATEGLINIDE) & 324 & 324 & 324 & 324 & 324 & 324 \\
\hline Polymer & 162.5 & 227.5 & 292.5 & 162.5 & 227.5 & 292.5 \\
\hline Dicalcium phoshphate & 144 & 79 & 14 & 144 & 79 & 14 \\
\hline Mg.Stearate & 13 & 13 & 13 & 13 & 13 & 13 \\
\hline Aerosil 200 & 6.5 & 6.5 & 6.5 & 6.5 & 6.5 & 6.5 \\
\hline TOTAL & $650 \mathrm{mg}$ & $650 \mathrm{mg}$ & $650 \mathrm{mg}$ & $650 \mathrm{mg}$ & $650 \mathrm{mg}$ & $650 \mathrm{mg}$ \\
\hline
\end{tabular}

\section{RESULTS}

Interaction between drug and wax was checked by FT-IR Spectroscopy.

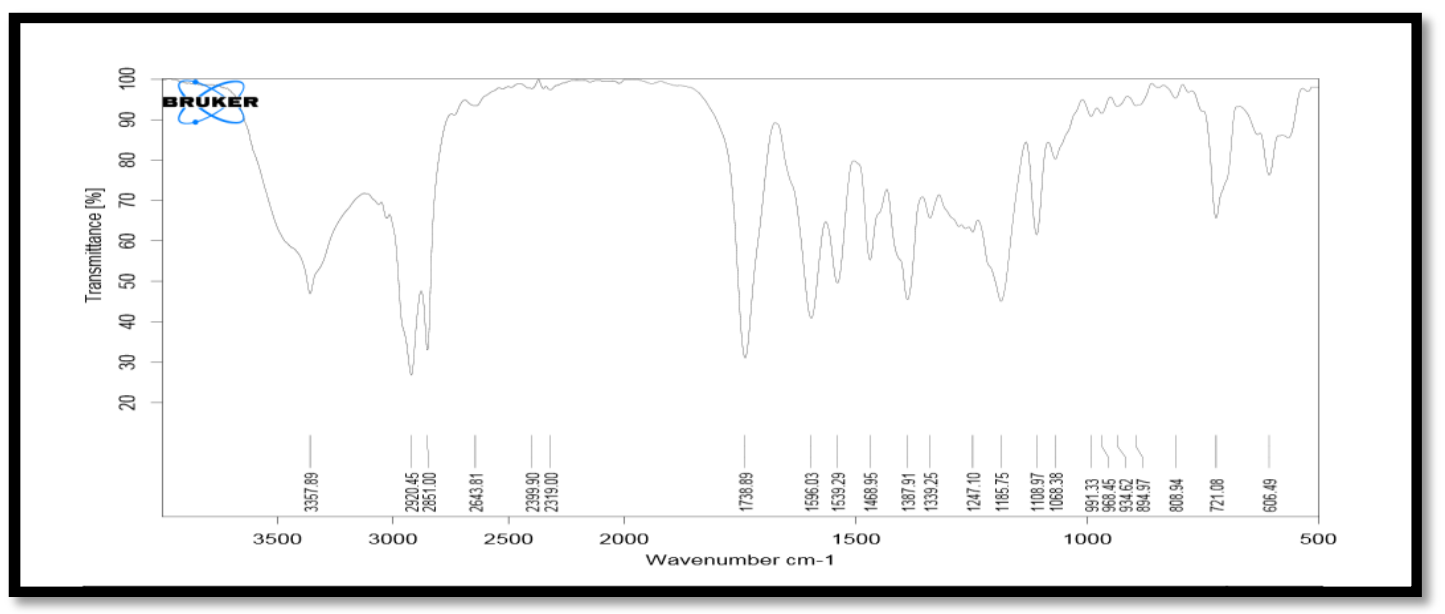

Figure 2: IR Spectrum of all excipients \& Nateglinide

\section{DIRECT COMPRESSION}

Table 2: Pre-compression parameters of batch F1-F6 (Direct compression)

\begin{tabular}{|c|c|c|c|c|c|}
\hline $\begin{array}{c}\text { Formulatio } \\
\mathbf{n}\end{array}$ & $\begin{array}{c}\text { Angle of } \\
\left.\text { Repose }^{(}\right) \\
(\mathbf{n}=\mathbf{3}) \pm \text { SD }\end{array}$ & $\begin{array}{c}\text { Loose Bulk } \\
\text { Density } \\
(\mathbf{g} / \mathbf{c m 3}) \pm \mathbf{S D}\end{array}$ & $\begin{array}{c}\text { Tapped Bulk } \\
\text { Density } \\
(\mathbf{g} / \mathbf{c m 3}) \pm \text { SD }\end{array}$ & $\begin{array}{c}\text { Hausners } \\
\text { Ratio } \pm \text { SD }\end{array}$ & $\begin{array}{c}\text { Carr's Index } \\
(\%) \pm \text { SD }\end{array}$ \\
\hline F1 & $30.81 \pm 0.90$ & $0.43 \pm 0.030$ & $0.53 \pm 0.036$ & $1.23 \pm 0.052$ & $18.86 \pm 0.77$ \\
\hline F2 & $29.75 \pm 0.81$ & $0.44 \pm 0.034$ & $0.56 \pm 0.028$ & $1.27 \pm 0.060$ & $21.40 \pm 0.25$ \\
\hline F3 & $29.19 \pm 0.68$ & $0.46 \pm 0.028$ & $0.57 \pm 0.052$ & $1.24 \pm 0.031$ & $19.29 \pm 0.16$ \\
\hline F4 & $28.40 \pm 0.74$ & $0.47 \pm 0.019$ & $0.58 \pm 0.043$ & $1.23 \pm 0.047$ & $18.98 \pm 0.29$ \\
\hline F5 & $30.18 \pm 0.65$ & $0.45 \pm 0.043$ & $0.56 \pm 0.042$ & $1.24 \pm 0.063$ & $19.64 \pm 0.45$ \\
\hline F6 & $32.39 \pm 1.02$ & $0.46 \pm 0.035$ & $0.57 \pm 0.051$ & $1.23 \pm 0.058$ & $19.29 \pm 0.87$ \\
\hline
\end{tabular}

Table 3: Post-compression parameters of batch F1-F6 (Direct compression)

\begin{tabular}{|c|c|c|c|c|c|}
\hline Formulation & $\begin{array}{c}\text { Hardness } \\
\left(\mathbf{K g} / \mathbf{C m}^{\mathbf{2}} \mathbf{\mathbf { ~ } \mathbf { S D }}\right.\end{array}$ & $\begin{array}{c}\text { Friability } \\
\mathbf{( \% )}\end{array}$ & $\begin{array}{c}\text { Weight variation(mg) } \\
\mathbf{\mathbf { \% } D}\end{array}$ & $\begin{array}{c}\text { Drug Content(\%) } \\
\mathbf{\pm S D}\end{array}$ & $\begin{array}{c}\text { Swelling } \\
\text { index }(\boldsymbol{\%})\end{array}$ \\
\hline F1 & $6.3 \pm 0.60$ & 0.23 & $649 \pm 5.12$ & $98.51 \pm 0.60$ & 10.1 \\
\hline F2 & $6.5 \pm 0.28$ & 0.21 & $650 \pm 4.94$ & $99.12 \pm 0.42$ & 25.6 \\
\hline F3 & $6.4 \pm 0.40$ & 0.19 & $648 \pm 6.39$ & $99.82 \pm 0.48$ & 37.9 \\
\hline F4 & $6.5 \pm 0.71$ & 0.17 & $650 \pm 5.38$ & $99.54 \pm 0.71$ & 24.4 \\
\hline F5 & $6.4 \pm 0.80$ & 0.14 & $649 \pm 4.29$ & $99.89 \pm 0.38$ & 41.9 \\
\hline F6 & $6.4 \pm 0.58$ & 0.13 & $649 \pm 4.35$ & $99.64 \pm 0.66$ & 54.6 \\
\hline
\end{tabular}


Table 4: In-Vitro Drug Release data of F1 to F6

\begin{tabular}{|c|c|c|c|c|c|c|}
\hline \multirow{2}{*}{ Time (h) } & \multicolumn{7}{|c|}{ In-Vitro Drug Release at $37^{0} \pm 0.5^{\circ} \mathrm{C}, 50$ RPM } \\
\cline { 2 - 7 } & F1 & F2 & F3 & F4 & F5 & 0 \\
\hline $\mathbf{0}$ & 0 & 0 & 0 & 2.5 & 3.13 & 0.85 \\
\hline $\mathbf{1}$ & 3.11 & 2.63 & 2.34 & 8.38 & 6.43 & 1.18 \\
\hline $\mathbf{2}$ & 10.26 & 11.13 & 5.6 & 12.27 & 8.57 & 2.1 \\
\hline $\mathbf{3}$ & 17.22 & 14.82 & 9.32 & 18.53 & 12.38 & 4.17 \\
\hline $\mathbf{4}$ & 26.62 & 17.21 & 13.53 & 22.64 & 14.75 & 6.42 \\
\hline $\mathbf{5}$ & 30.39 & 18.33 & 17.11 & 29.52 & 19.37 & 9.1 \\
\hline $\mathbf{6}$ & 33.67 & 21.46 & 21.34 & 33.77 & 23.56 & 11.4 \\
\hline $\mathbf{7}$ & 34.28 & 24.52 & 24.66 & 36.5 & 27.54 & 13.34 \\
\hline $\mathbf{8}$ & 39.88 & 26.67 & 27.43 & 39.71 & 30.64 & 15.72 \\
\hline $\mathbf{9}$ & 42.31 & 29.33 & 29.16 & 42.35 & 34.77 & 17.36 \\
\hline $\mathbf{1 1}$ & 47.37 & 31.74 & 31.42 & 43.66 & 41.36 & 19.83 \\
\hline $\mathbf{1 2}$ & 51.32 & 41.03 & 33.48 & 44.15 & 45.87 & 21.43 \\
\hline
\end{tabular}
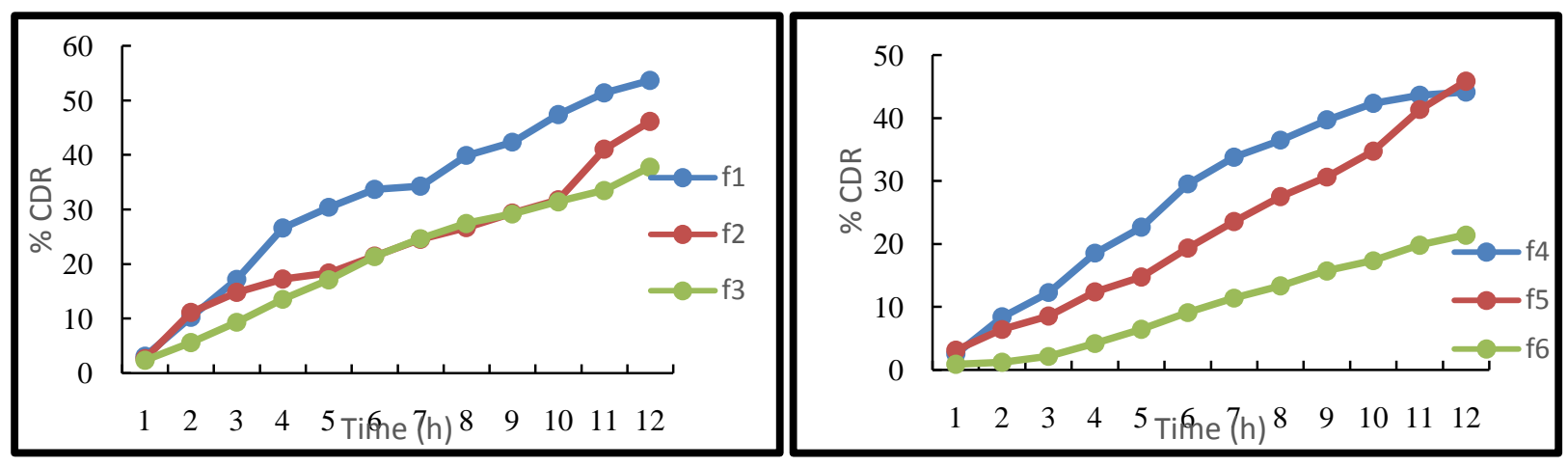

Figure 3: In-VitroIn-Vitro Drug Release of formulations F1 to F6

\section{CO-PROCESSING}

Table 5: Pre-compression parameters of batch F7-F12 (Co-processing)

\begin{tabular}{|c|c|c|c|c|c|}
\hline $\begin{array}{c}\text { Formulatio } \\
\mathbf{n}\end{array}$ & $\begin{array}{c}\text { Angle of } \\
\left.\text { Repose }^{(}\right) \\
(\mathbf{n}=\mathbf{3}) \pm \text { S.D }\end{array}$ & $\begin{array}{c}\text { Loose Bulk } \\
\text { Density } \\
(\mathbf{g} / \mathbf{c m 3}) \pm \text { S.D }\end{array}$ & $\begin{array}{c}\text { Tapped Bulk } \\
\text { Density } \\
(\mathbf{g} / \mathbf{c m 3}) \pm \text { S.D }\end{array}$ & $\begin{array}{c}\text { Hausners Ratio) } \\
\pm S . D\end{array}$ & $\begin{array}{c}\text { Carr's Index } \\
(\boldsymbol{\%})) \pm S . D\end{array}$ \\
\hline F7 & $31.22 \pm 0.84$ & $0.45 \pm 0.021$ & $0.56 \pm 0.054$ & $1.24 \pm 0.061$ & $19.64 \pm 0.93$ \\
\hline F8 & $33.04 \pm 0.32$ & $0.46 \pm 0.037$ & $0.55 \pm 0.044$ & $1.19 \pm 0.052$ & $16.35 \pm 0.61$ \\
\hline F9 & $29.65 \pm 0.55$ & $0.44 \pm 0.024$ & $0.54 \pm 0.041$ & $1.22 \pm 0.047$ & $22.72 \pm 0.45$ \\
\hline F10 & $27.25 \pm 0.74$ & $0.47 \pm 0.029$ & $0.56 \pm 0.063$ & $1.20 \pm 0.084$ & $16.18 \pm 0.77$ \\
\hline F11 & $28.58 \pm 0.86$ & $0.45 \pm 0.027$ & $0.54 \pm 0.032$ & $1.20 \pm 0.063$ & $16.66 \pm 0.45$ \\
\hline F12 & $31.20 \pm 0.40$ & $0.46 \pm 0.035$ & $0.57 \pm 0.061$ & $1.23 \pm 0.038$ & $19.29 \pm 0.57$ \\
\hline
\end{tabular}

Table 6: Post-compression parameters of batch F7-F12

\begin{tabular}{|c|c|c|c|c|c|}
\hline Formulation & $\begin{array}{c}\text { Hardness } \\
\left(\mathbf{K g} / \mathbf{C m}^{\mathbf{2}}\right) \mathbf{+ S D}\end{array}$ & Friability (\%) & Weight variation(mg) \pm SD & $\begin{array}{c}\text { Drug } \\
\text { Content(\%) } \pm \text { SD }\end{array}$ & $\begin{array}{c}\text { Swelling } \\
\text { index }(\boldsymbol{\%})\end{array}$ \\
\hline F7 & $6.7 \pm 0.27$ & 0.14 & $650 \pm 4.08$ & $99.85 \pm 0.12$ & 12.3 \\
\hline F8 & $6.2 \pm 0.84$ & 0.12 & $648 \pm 3.97$ & $99.56 \pm 0.34$ & 23.5 \\
\hline F9 & $6.4 \pm 0.79$ & 0.10 & $649 \pm 6.41$ & $98.17 \pm 0.87$ & 39.6 \\
\hline F10 & $6.3 \pm 0.61$ & 0.15 & $648 \pm 5.92$ & $97.9 \pm 0.65$ & 23.7 \\
\hline F11 & $6.6 \pm 0.85$ & 0.15 & $649 \pm 7.81$ & $99.74 \pm 0.14$ & 43.6 \\
\hline F12 & $6.7 \pm 0.93$ & 0.13 & $650 \pm 4.16$ & $98.89 \pm 0.10$ & 61.8 \\
\hline
\end{tabular}


Table 7: In-Vitro Drug Release datas of F7 to F12

\begin{tabular}{|c|c|c|c|c|c|c|}
\hline \multirow{2}{*}{ Time (h) } & \multicolumn{6}{|c|}{ In-Vitro Drug Release at $37^{\circ} \pm 0.5^{\circ} \mathrm{C}, 50 \mathrm{RPM}$} \\
\hline & F7 & F8 & F9 & F10 & F11 & F12 \\
\hline $\mathbf{0}$ & 0 & 0 & 0 & 0 & 0 & 0 \\
\hline 1 & 10.10 & 4.55 & 3.67 & 11.60 & 15.86 & 1.94 \\
\hline 2 & 18.47 & 11.43 & 11.12 & 23.72 & 27.06 & 3.83 \\
\hline 3 & 28.33 & 19.24 & 16.55 & 33.47 & 34.12 & 5.96 \\
\hline 4 & 37.44 & 25.35 & 22.80 & 37.56 & 41.37 & 8.1 \\
\hline 5 & 43.60 & 36.76 & 27.49 & 42.33 & 44.16 & 9.73 \\
\hline 6 & 48.72 & 41.40 & 31.36 & 49.13 & 48.43 & 13.43 \\
\hline 7 & 54.43 & 48.69 & 35.21 & 57.15 & 51.20 & 16.6 \\
\hline 8 & 63.34 & 53.23 & 41.17 & 61.48 & 55.63 & 19.58 \\
\hline 9 & 71.56 & 59.92 & 44.83 & 65.52 & 59.55 & 22.67 \\
\hline 10 & 77.89 & 63.53 & 49.59 & 68.84 & 62.74 & 24.14 \\
\hline 11 & 85.54 & 70.47 & 52.58 & 72.48 & 65.38 & 28.45 \\
\hline 12 & 87.92 & 75.01 & 56.71 & 83.20 & 66.47 & 33.32 \\
\hline
\end{tabular}
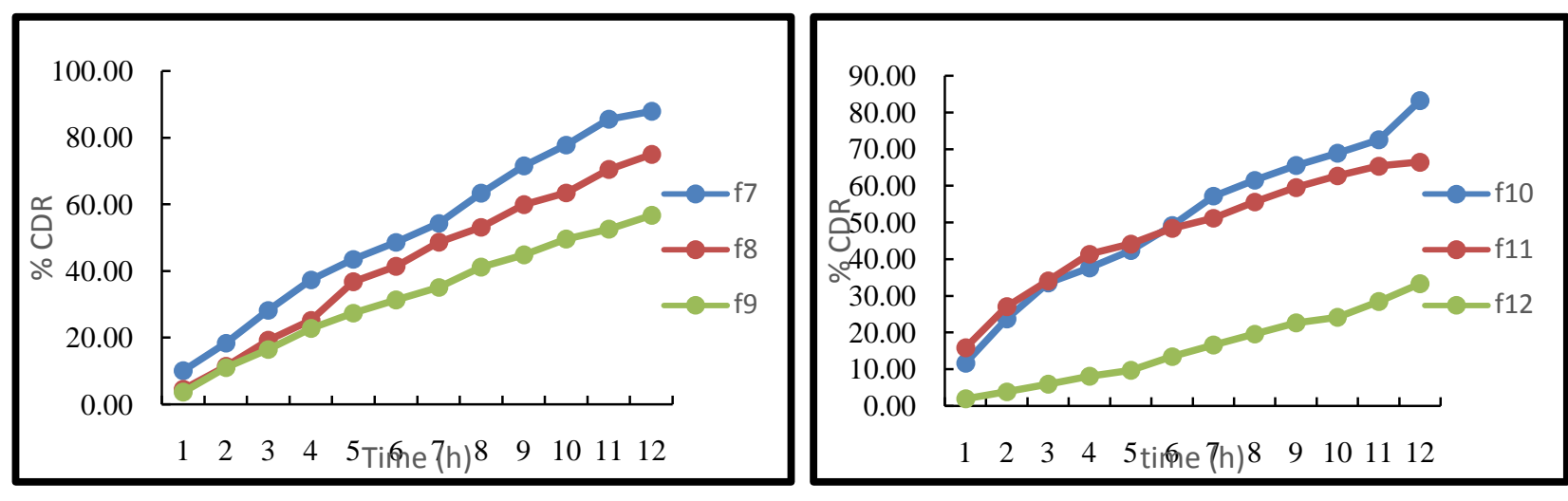

Fig 4: In-VitroIn-Vitro Drug Release of formulations F7 to F12

\section{MELT GRANULATION}

Table 8: Pre-compression parameters of batch F13-F18

\begin{tabular}{|c|c|c|c|c|c|}
\hline Formulation & $\begin{array}{c}\text { Angle of Repose }\left(^{\circ}\right) \\
(\mathbf{n}=\mathbf{3})\end{array}$ & $\begin{array}{c}\text { Loose Bulk Density } \\
(\mathbf{g} / \mathbf{c m 3})\end{array}$ & $\begin{array}{c}\text { Tapped Bulk Density } \\
(\mathbf{g} / \mathbf{c m 3})\end{array}$ & $\begin{array}{c}\text { Hausners } \\
\text { Ratio }\end{array}$ & $\begin{array}{c}\text { Carr's Index } \\
(\%)\end{array}$ \\
\hline F13 & $18.20 \pm 0.88$ & $0.59 \pm 0.030$ & $0.66 \pm 0.045$ & $1.12 \pm 0.06$ & $10.06 \pm 0.72$ \\
\hline F14 & $19.87 \pm 0.43$ & $0.67 \pm 0.028$ & $0.72 \pm 0.032$ & $1.07 \pm 0.05$ & $6.94 \pm 0.93$ \\
\hline F15 & $17.07 \pm 1.01$ & $0.58 \pm 0.034$ & $0.64 \pm 0.037$ & $1.10 \pm 0.07$ & $9.37 \pm 0.60$ \\
\hline F16 & $19.41 \pm 0.98$ & $0.62 \pm 0.042$ & $0.68 \pm 0.029$ & $1.09 \pm 0.07$ & $8.88 \pm 0.43$ \\
\hline F17 & $19.43 \pm 0.79$ & $0.57 \pm 0.011$ & $0.62 \pm 0.050$ & $1.08 \pm 0.09$ & $8.09 \pm 0.38$ \\
\hline F18 & $16.65 \pm 0.47$ & $0.57 \pm 0.052$ & $0.63 \pm 0.064$ & $1.19 \pm 0.04$ & $9.52 \pm 0.62$ \\
\hline
\end{tabular}

Table 9: Post-compression parameters of batch F13-F18

\begin{tabular}{|c|c|c|c|c|c|}
\hline Formulation & $\begin{array}{c}\text { Hardness } \\
\left(\mathbf{K g} / \mathbf{C m}^{\mathbf{2}}\right) \mathbf{\pm S D}\end{array}$ & Friability (\%) & Weight variation(mg) $\mathbf{S S D}$ & $\begin{array}{c}\text { Drug Content(\%) } \\
\mathbf{\pm S D}\end{array}$ & $\begin{array}{c}\text { Swelling } \\
\text { index }(\boldsymbol{\%})\end{array}$ \\
\hline F13 & $6.5 \pm 0.38$ & 0.13 & $650 \pm 4.65$ & $99.89 \pm 0.50$ & 22.9 \\
\hline F14 & $6.8 \pm 0.61$ & 0.14 & $647 \pm 5.29$ & $99.89 \pm 0.76$ & 36.4 \\
\hline F15 & $6.3 \pm 0.29$ & 0.13 & $648 \pm 3.39$ & $99.89 \pm 0.47$ & 42.3 \\
\hline F16 & $6.4 \pm 0.51$ & 0.19 & $649 \pm 5.02$ & $99.89 \pm 0.58$ & 29.1 \\
\hline F17 & $6.6 \pm 0.27$ & 0.14 & $648 \pm 4.92$ & $99.64 \pm 0.60$ & 34.9 \\
\hline F18 & $6.2 \pm 0.74$ & 0.17 & $500 \pm 5.16$ & $99.85 \pm 0.34$ & 53.5 \\
\hline
\end{tabular}


Table 10: In-Vitro Drug Release datas of F13 to F18

\begin{tabular}{|c|c|c|c|c|c|c|}
\hline \multirow[b]{2}{*}{ Time (h) } & \multicolumn{6}{|c|}{ In-Vitro Drug Release at $37^{0} \pm 0.5^{\circ} \mathrm{C}, 50 \mathrm{RPM}$} \\
\hline & F13 & F14 & F15 & F16 & F17 & F18 \\
\hline $\mathbf{0}$ & 0 & 0 & 0 & 0 & 0 & 0 \\
\hline 1 & 11.47 & 11.06 & 8.43 & 9.12 & 10.21 & 7.42 \\
\hline 2 & 24.42 & 19.65 & 14.96 & 11.36 & 16.80 & 11.15 \\
\hline 3 & 40.62 & 33.04 & 23.25 & 20.82 & 24.15 & 17.14 \\
\hline 4 & 48.99 & 38.47 & 28.74 & 36.74 & 28.68 & 20.96 \\
\hline 5 & 58.79 & 46.07 & 33.62 & 43.06 & 34.49 & 25.41 \\
\hline 6 & 67.13 & 53.34 & 38.39 & 50.61 & 41.06 & 28.94 \\
\hline 7 & 72.47 & 60.62 & 43.53 & 62.21 & 47.44 & 32.12 \\
\hline 8 & 79.05 & 66.07 & 48.68 & 76.15 & 51.88 & 36.06 \\
\hline 9 & 84.05 & 70.84 & 52.42 & 83.90 & 59.34 & 39.15 \\
\hline 10 & 90.36 & 75.05 & 56.43 & 89.97 & 64.89 & 42.88 \\
\hline 11 & 92.13 & 82.61 & 62.12 & 91.25 & 66.21 & 48.25 \\
\hline 12 & 94.55 & 86.54 & 68.32 & 92.10 & 71.45 & 54.13 \\
\hline
\end{tabular}
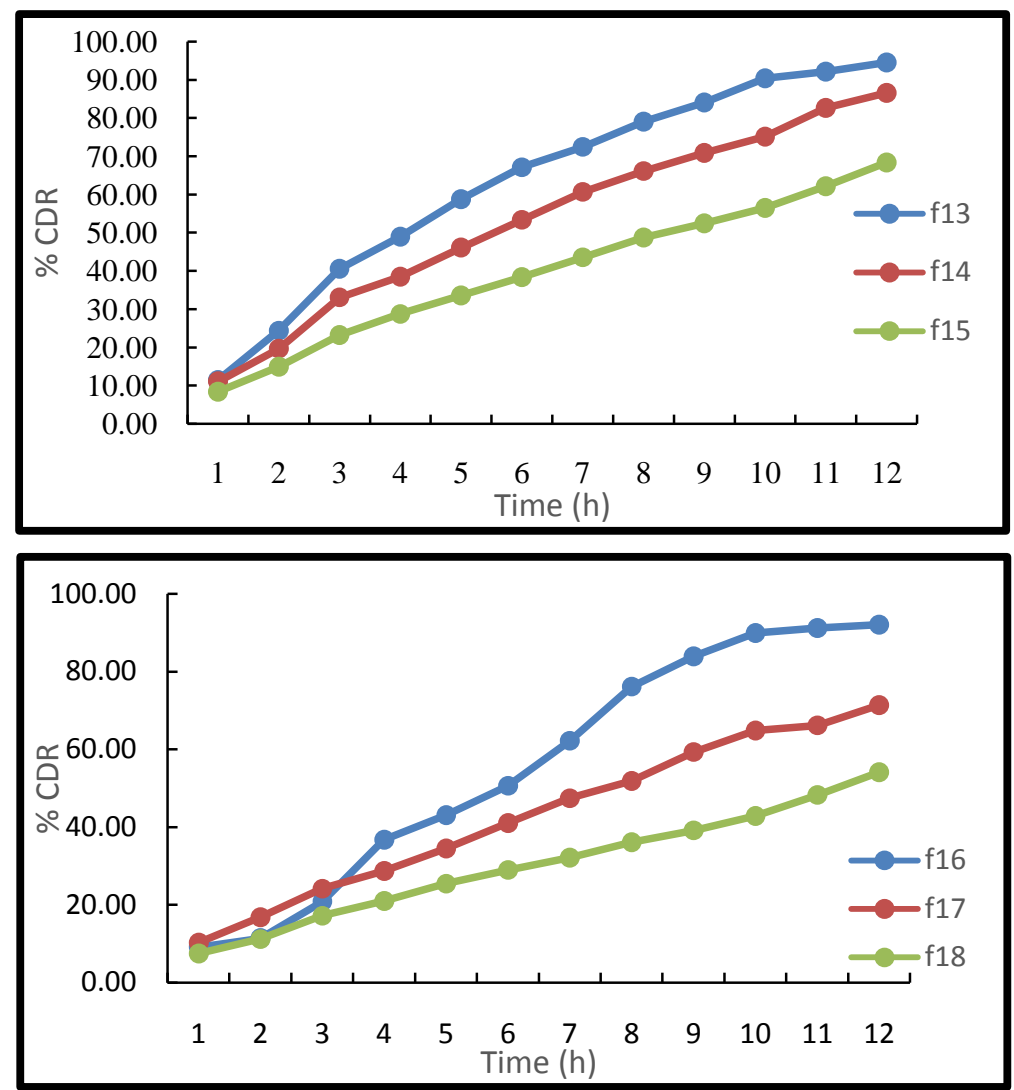

Fig 5: In-VitroIn-Vitro Drug Release of formulations F13 to F18

\section{DISCUSSION:}

The study showed that Precirol ATO 5 is an appropriate waxy matrix former for sustained release of low watersoluble drug such as Nateglinide.MG technique fits completely into the predetermined parameter and criteria whereas, other two techniques lack behind.
Sustained release profiles can be achieved by any of three methods using Precirol ATO 5 at higher proportion but MG technique achieved $12 \mathrm{~h}$ release profile with lesser amount of was as compare to other methods. Formulation F13 was given $94.55 \%$ drug release in $12 \mathrm{~h}$ which was optimizing batch. 


\section{REFERENCES:}

1. Gudsoorkar VR, Rambhau D. "Influence variable of process one standard drug release from disintegrating sustained release Ibuprofen beads" Eastern Pharmacist,1997, 40(2), 111-113.

2. Siddique S, Bose A, Khanam, J. "Modulation of drug (metoprolol succinate) release by inclusion of hydrophobic polymer in hydrophilic matrix."Drug Dev Ind Pharm2011, 37(9), 1016-1025.

3. Deore RK, Kavitha K, Tamizhmani TG. "Preparation and evaluation of sustained release matrix tablets of tramadol hydrochloride using glyceryl palmitostearate."Trop J Pharm Res, 2010, 9(3), 275-281.

4. Heng WS, Wong TW. "Investigation of melt agglomeration process with hydrophobic binder in combination with sucrose stearate". Eur J Pharm Sci, 2003, 19(5), 381-393.

5. Bodmeier RA, Swarbrick J, Boylan JC. Eds. Encyclopedia of pharmaceutical technology. $2^{\text {nd }}$ ed. Vol. III, New York: Marcel Dekker lnc; 1998.

6. Hamdani J, Moes AJ, Amighi K. "Physical \& thermal characterization of Precirol \& Compritol as lipophilic glycerides used for the preparation of controlled release matrix pellets."Int J Pharm,2003, 260, 47-57.

7. Zang YE, Schwartz JB. "Melt granulation and heat treatment for wax matrix-controlled drug release."Drug Dev Ind Pharm, 2003, 29, 131-138

8. Hamdani J, Moes AJ, Amighi K. "Development and evaluation of prolonged release pellets obtained by melt pelletization process."Int J Pharm, 2002, 245, 167-177.

9. Lloanusi NO, Schwartz JB. "The effect of wax on compaction of microcrystalline cellulose beads made by extrusion and spheronization."Drug Dev Ind Pharm, 1998, 24, 37-44.

10. Jannin V, Cuppok Y. "Hot-melt coating with lipid excipient."Int J Pharm, 2013, 457, 480-487.

11. Mahaparale PR, Kasture PV, Deshmukh SS et al. "Sustained release matrices of Metoprolol succinate using Compritol 888 ATO and Precirol ATO 05". J Pharm Res, 2006, 5, 10-14.

12. Patere SN, Desai NS, Jain AK et al. "Compritol ${ }^{\circledR} 888$ ATO a lipid excipient for sustained release of highly water soluble active: formulation, scale-up and IVIVC study."Current Drug Del,2013, 10, 548-556.

13. Yubing Z, Qian Z, Cuixia Y, Junlin C, Yunfang H, Jianjun $\mathrm{Z}$, Lu Y and Jianhua M, "Relative fasting bioavailability of two formulations of nateglinide $60 \mathrm{mg}$ in healthy male chinese volunteers: anopen-label, randomized-sequence, single-dose, two-waycrossover study." Clinical Therapeutics, 2012, 34, 1505-1510.

14. Kajjari PB, Manjeshwar LS and Aminabhavi TM, "Novel blend microspheres of cellulose triacetate and bee wax for the controlled release of nateglinide." Journal of Industrial and Engineering Chemistry, 2014, 20, 397-404.

15. Bell PM, Cuthbertson J, Patterson S and O'Harte FPM, "Additive hypoglycaemic effect of nateglinide and exogenous glucagon-like peptide-1 in type 2 diabetes."Diabetes Research and Clinical Practice, 2011, 91, 68 - 70.

16. Tanaka N, Imai $\mathrm{K}$ and Ueda $\mathrm{S}$. "Development of novel sustained-release system, disintegration-controlled matrix tablet (DCMT) with solid dispersion granules of nilvadipine" J. Cont. Rel., 2005, 108, 386-395.

17. Obaidat A.A. and Obaidat R.M. "Controlled release of tramadol tydrochloride frommatrices prepared using glyceryl behenate" Eur. J. Biopharm. Pharmacokin., 2001; 52(2); 231235.

18. Brabander C. D, Gortz J P and Berlo J A. "Bioavaliability of ibuprofen from matrix mini-tablets based on a mixture of starch and microcrystalline wax" Int. J. Pharma.,2000, 208, 81-86.

19. Bhalekar M R, Madgulkar A R and Wable N D. "Statistical optimization of sustained release venlafaxine $\mathrm{HCl}$ wax matrix tablet" Ind. J. Pharma. Sci., 2008, 70(4), 472-476.

20. Vyas J, Upadhyay $\mathrm{T}$ and Vyas $\mathrm{H}$. "Development and evaluation of bilayered gastro-retentive tablet containing metformin $\mathrm{HCl} \mathrm{SR}$ and pioglitazonehcl IR " J. drug del. thera. 2013, 3(5), 58-61.

21. Verhoeven E, Beer T R M and Mooter G V"Influence of formulation and process parameters on the release characteristics of ethylcellulose sustained-release minimatrices produced by hot-melt extrusion" Eur. J. Biopharm. Pharmacokin., 2008; 69, 312-319.

22. Ramana G and Roy D."Formulation and evaluation of channeling agent on release pattern of ambroxol hydrochloride from HPMC K4M matrix tablets" Asian $J$. Pharma. Hea. Sci., 2012, 2(4), 467-471.

23. Masareddy R and Kendalakar P V "Effect of polymers as matrix system in formulation of sustained release theophylline matrix tablet" Int. J Pharm. Pharmaceu. Sci.,2012, 4(4), 409414.

24. Passerini N, Perissutti and Albetini B, "Controlled release of verapamil hydrochloride from waxy microparticles prepared by spray congealing" J. Cont. Rel. 2003, 88; 263-275.

25. Brabander $\mathrm{C}$, Vervaet $\mathrm{C}$ and Fiermans L. "Matrix minitablets based on starch:microcrystalline wax mixtures" Int. J. Pharma., 2000, 199, 195-203.

26. Nnadi, Okeke C and Amechi A. "Design and evaluation of sustained release potential of diclofenac potassium contained in beeswax matrix" Int. J. Res. In Med. Health Sci. 2013, 1, 31-36.

27. Dankaisorn W and Phaechamud T. "Sustained release of propranolol $\mathrm{HCl}$ using matrix granule comprising wax and polymers" Res. J. Pharma. Bio. Chem. Sci. 2011, 2(1), 158166.

28. Shady M, Amin A and Gawad N. "Comparative study on the different techniques for the preparation of sustained-release hydrophobic matrices of a highly water-soluble drug " Drug Disc Thera. 2010, 4(6), 484-492.

29. Cheboyina S and Wyandt C. "Wax-based sustained release matrix pellets prepared by a novel freeze pelletization technique II. In vitro drug release studies and release mechanisms" Int. J. Pharma., 2008, 359, 167-173.

30. Qian Li F, Hong H J and Deng J X. "In vitro controlled release of sodium ferulate from Compritol 888 ATO-based matrix tablets" Int. J. Pharma., 2006, 324, 152-157. 